Research Paper

\title{
Mid-infrared T-shaped photonic crystal waveguide for optical refractive index sensing
}

\author{
Mirbek Turduev ${ }^{\mathrm{a}, *}$, Ibrahim H. Giden ${ }^{\mathrm{b}}$, Ceren Babayiğit ${ }^{\mathrm{a}}$, Zeki Hayran ${ }^{\mathrm{b}}$, Emre Bor ${ }^{\mathrm{a}, \mathrm{b}}$, \\ Çiçek Boztuğ ${ }^{\mathrm{a}}$, Hamza Kurtt ${ }^{\mathrm{b}}$, Kestutis Staliunas ${ }^{\mathrm{c}, \mathrm{d}}$ \\ a Photonic Networks Research Group, TED University, Department of Electrical and Electronics Engineering, Ankara, Turkey \\ ${ }^{\mathrm{b}}$ Nanophotonics Research Group, TOBB University of Economics and Technology, Department of Electrical and Electronics Engineering, Ankara, Turkey \\ c DONLL, Departament de Física i Enginyeria Nuclear, Universitat Politècnica de Catalunya (UPC), Terrassa, Spain \\ d Institució Catalana de Recercai Estudis Avançats (ICREA), Barcelona, Spain
}

\section{A R T I C L E I N F O}

\section{Article history:}

Received 22 October 2016

Received in revised form 31 January 2017

Accepted 1 February 2017

Available online 3 February 2017

\section{Keywords:}

Photonic crystals

Bio-chemical sensing

Slotted waveguides

Label free optical sensors

Refractive index based sensors

\begin{abstract}
A B S T R A C T
In this paper, we propose and design a new type of an integrated optical sensor that performs sensing in a wide wavelength range corresponding to mid-infrared (mid-IR) spectrum. By engineering the structural parameters of square-lattice photonic crystal (PC) slab incorporated with a T-shaped air-slot, strong light confinement and interaction with the analytes are assured. Numerical analyses in the time and frequency domain are conducted to determine the structural parameters of the design. The direct interaction between the slot waveguide mode and the analyte infiltrated into the slot gives rise to highly sensitive refractive index sensors. The highest sensitivity of the proposed T-slotted PC sensor is $1040 \mathrm{~nm} / \mathrm{RIU}$ within the range of analytes' refractive indices $n=1.05-1.10$, and the overall sensitivity corresponding to the higher refractive index range of $n=1.10-1.30$ is around $500 \mathrm{~nm} / \mathrm{RIU}$. Moreover, for a realistic PC slab structure, we determined an average refractive index sensitivity of $530 \mathrm{~nm} / \mathrm{RIU}$ within the range of $\mathrm{n}=1.10-1.25$ and an average sensitivity of $390 \mathrm{~nm} / \mathrm{RIU}$ within the range of $\mathrm{n}=1.00-1.30$. Furthermore, we speculate on the possible approach for the fabrication and the optical characterization of the device. The assets of the device include being compact, having a feasible measurement and fabrication technique, and possessing label-free sensing characteristic. We expect that the presented work may lead to the further development of the mid-IR label-free biochemical sensor devices for detection of various materials and gases in the near future.
\end{abstract}

(c) 2017 Elsevier B.V. All rights reserved.

\section{Introduction}

Within the last decades, photonic sensors have been an intensive research subject since a tremendous demand for sensing platforms has occurred especially in the detection of biological, chemical/bio-chemical and nuclear agents [1]. Different types of photonic devices have been proposed as an optical sensor such as microring resonators [2], plasmonic metamaterials [3], and interferometers [4]. The recent developments in the photonic technologies allow realizing high-performance sensing devices in terms of sensor sensitivity, limit of detection and response time. To produce commercial and low-cost photonic bio-chemical sensors, the following properties should be satisfied; reliability, portability, compactness and the fabrication feasibility with available CMOS and Silicon-on-insulator (SOI) technologies. In general, the oper-

\footnotetext{
* Corresponding author.

E-mail address: mirbek.turduev@tedu.edu.tr (M. Turduev).
}

ational principle of optical sensors is based on different sensing mechanisms such as spectral [5], phase-shift [6], and amplitude sensing [7]. Specifically, the detection criterion in spectral sensing case is based on the rate of resonance wavelength shift $(\Delta l)$ with respect to the change in the refractive index $(\Delta n)$, so that the sensitivity parameter is defined as $S=\Delta \lambda / \Delta n$ with the corresponding unit of $\mathrm{nm} / \mathrm{RIU}$. Sensors based on the spectral sensing are designed such that they support waveguide or cavity modes. On the other hand, phase-shift and amplitude sensing mechanisms are based on the detection of phase and amplitude changes of the light detected at the output, which depends on the refractive index of the analyte.

As a state-of-the-art sensor technology, photonic crystals (PCs) [8] can be nominated as a potential candidate for various optical sensing applications due to their highly dispersive nature and high sensitivity to the material index variations. A variety of PC based sensors with the following sensing mechanisms have been realized: Refractive index (RI) modification [9,10], optical absorption modification [11], opto-mechanical modification [12], and the modification in the non-linear effects [13]. RI-based sensors 
are widely-studied class of PC sensors among above-mentioned sensing mechanisms. Two fundamental principles are employed in RI-based sensors; homogeneous sensing due to effective index modifications of the covered medium $[14,15]$ and surface sensing depending on immobilized target molecules on the surface. In the latter case, a thickness change occurred in the vicinity of the sensor surface because of the captured bio-molecular layer, which in turn induces an effective refractive index change [16]. Apart from PC-based sensors, micro-structured optical fibers have also been implemented for different applications such as strain [17] and biochemical sensing [18].

In this study, a T-slotted PC slab waveguide is designed by introducing inner line-defects and an air T-slot in perfect squarelattice air holes. The optical sensitivity of the proposed PC sensor is investigated in both spectral and spatial domains based on the refractive index variation of infiltrated analyte. Several advantages may emerge due to using an air-slotted section: One is the strong modal confinement achieved by the slotted PC waveguides, which enables strong light-matter interaction with the analysed samples $[19,20]$. The slotted architecture may also promote single-mode optical guiding that avoids inter-mode crosstalk arising from multimode input beam [21]. The dimensions of the sensor system is arranged to operate at mid-infrared (MIR) wavelengths. The MIR regime gathered great interests especially for sensing applications since the absorption resonances - so-called vibrational "fingerprints" - of many bio-chemical molecules are involved in this spectral range [22-24].

The paper is organized as follows: in Section 2, the schematic of designed PC slab sensor and its operating principles are explored. Then, temporal and spectral analyses of the proposed PC based sensor are presented in Section 3. Next, the possible fabrication procedure of these structures is given in Section 4, together with a transmission measurement setup as a potential platform to characterize these slotted PC waveguides. Finally, the summary of the study is provided in Conclusion section.

\section{The design approach and principle of operation}

The MIR wavelength range corresponds to $2-20 \mu \mathrm{m}$ and this spectral range is useful for biological and gas sensing applications [25]. It was reported in Ref. [26] that Si could be considered a suitable low-loss material at MIR wavelengths and thus, it could be utilized for the fabrication of passive integrated photonic devices. For this reason, the proposed PC slab sensor is designed to operate in the 2.0-2.5 $\mu \mathrm{m}$ window of MIR spectral range and realized on silicon-on-insulator (SIO) substrate by taking the advantage of the negligible refractive index variation of $\mathrm{Si}$ in this wavelength range. As a result, the material dispersion is neglected in the numerical calculations. The three-dimensional (3D) schematic of the designed PC-based sensor is shown in Fig. 1(a). In order to explore operational principle of proposed sensor device, it is important to clarify the crucial sections and geometrical parameters of the PC sensor design. The top-view of the proposed sensor is presented with colour-shadings of different regions in Fig. 1(b). As can be seen from the figure, the structure consists of three different operating sections: multimode guiding, sample detection, and filtering region. The multimode PC guiding region includes square lattice PC holes in dielectric background where wide line-defects are introduced that allow light to propagate inside PC waveguide with a strong volume confinement. Such type of a PC waveguide supports multimode guiding in certain wavelengths and the guided beam encounters interactions with the bio-chemical samples inside the T-slot, which will result in the peak frequency shifts in the transmitted light spectrum depending on the samples' refractive indices. By the help of the last section of the PC sensor platform, i.e. the slotted PC waveguide filtering region, the guided light is filtered to a single resonant mode at operating wavelengths. Since the resonance peaks are directly related to the material characteristics of infiltrated analytes, slight variations on their refractive indices may provide substantial resonance peaks' shifts in the transmission spectra. It should be noted that even though T-shaped slot introduced into the PC slab has already been discussed in one of our previous works [27], the sensing mechanism where the focusing capability of graded-index PC (GRIN PC) is utilized is rather different than the present study, where the guided multimodes are excited at the guiding region and filtered to a single mode at the filtering region.

The geometry of T-slot, where different samples will be infiltrated, is represented with its dimensions in Fig. 1(c). As can be seen from the figure, the structure is composed of Si having the refractive index of $n_{S i}=3.47$. The radii of the square lattice PC air holes in the designed structure are equal to $r=0.35 a$, where " $a$ " is the lattice constant. The periodicity is broken by introducing linedefects in guiding and filtering regions in order to create input and output waveguide channels. Corresponding widths of the in-/outchannels are equal to $\left\{w_{i n}, w_{\text {out }}\right\}=\{4 a, 2 a\}$. The structural parameters of $\mathrm{T}$-shaped slot within the detection and filtering regions are optimized to be $\left\{s_{x}, s_{y}, w_{\text {slot }}\right\}=\{0.8 a, 14.4 a, 0.5 a\}$, see Fig. 1 (c). The purpose of the introduced T-slot can be elucidated as follows: The vertical slot in detection region as in Fig. 1(c) operates as a feedback mechanism that enables strong wave-matter interaction within the T-shaped slot. The propagating modes that enter the Tslot are initially forced to a partial beam splitting in the transverse $y$-direction. Then, the splitted portion of beam reflects back from the vertical ends due to Fresnel reflection and interferes either constructively or destructively at the input of the horizontal (parallel to propagation direction) slot depending on the refractive indices of the analytes. The beam propagation path is illustrated with the red arrows in Fig. 1(b). As can be seen from Fig. 1(c), the transition between the vertical and horizontal slots is maintained with a smooth bending region with a bending radius of $R=1.35 a$ to increase the delivered power amount from the detection region to the filtering region. That condition makes a field enhancement in the resonance peaks' shift obtained at the output channel. Since the designed PC based sensor device is desired to operate in the MIR spectrum, the lattice constant is fixed to $a=500 \mathrm{~nm}$. Hence, the entire dimensions of proposed $2 \mathrm{D} / 3 \mathrm{D}$ sensor system are calculated to be $\left\{L_{x}, L_{y}\right\}=\{6.1 \mu \mathrm{m}, 7.0 \mu \mathrm{m}\}$.

\section{Spectral and spatial analyses of 2D slab photonic sensor}

PC based devices have gathered great attention within the last decades and pushed the "manipulation of light" ability to higher levels due to their underlying light confinement mechanism based on photonic bandgap phenomenon. Multiple scattering of light due to the periodically modulated dielectric constant of the PC leads to creation of photonic bandgaps, where light propagation is forbidden for certain frequency intervals. Thus, by introducing line- defects inside a periodic PC lattice, one can form optical waveguides with considerably low-loss [28]. Spectral properties of designed PC sensor can be examined separately for each specified region as in Fig. 1(b) using classical plane-wave expansion method [29]. Corresponding 2D band structures are calculated for transverse electric (TE) polarization, in which the magnetic field is perpendicular to the plane of periodicity. In this case, dispersion relations of an ideal square lattice 2D PC holes having radii of $r=175 \mathrm{~nm}$ are analyzed for TE polarization and the results are demonstrated Fig. 2(a). A photonic bandgap in the normalized frequency region of $0.1628-0.2456(a / \lambda)$ is calculated along the $\Gamma$ $\mathrm{X}$ direction (light propagation is in the $x$-direction). Introducing 

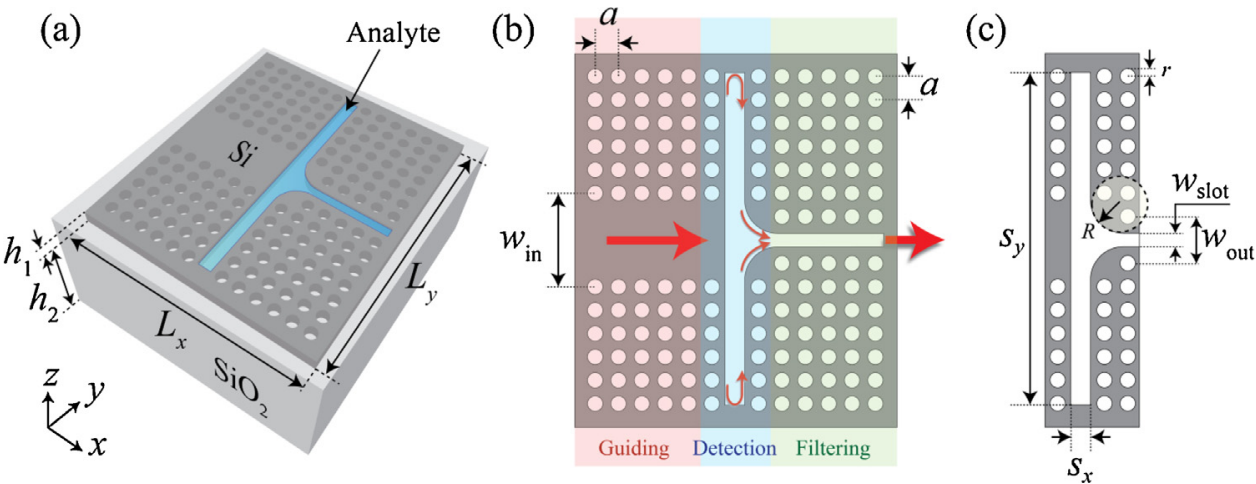

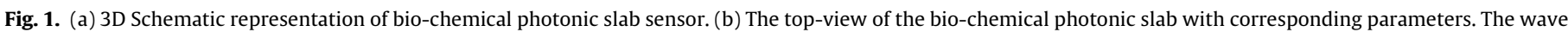
propagation within the slot is explored by arrows. (c) The structural parameters of the introduced T-shaped slot.
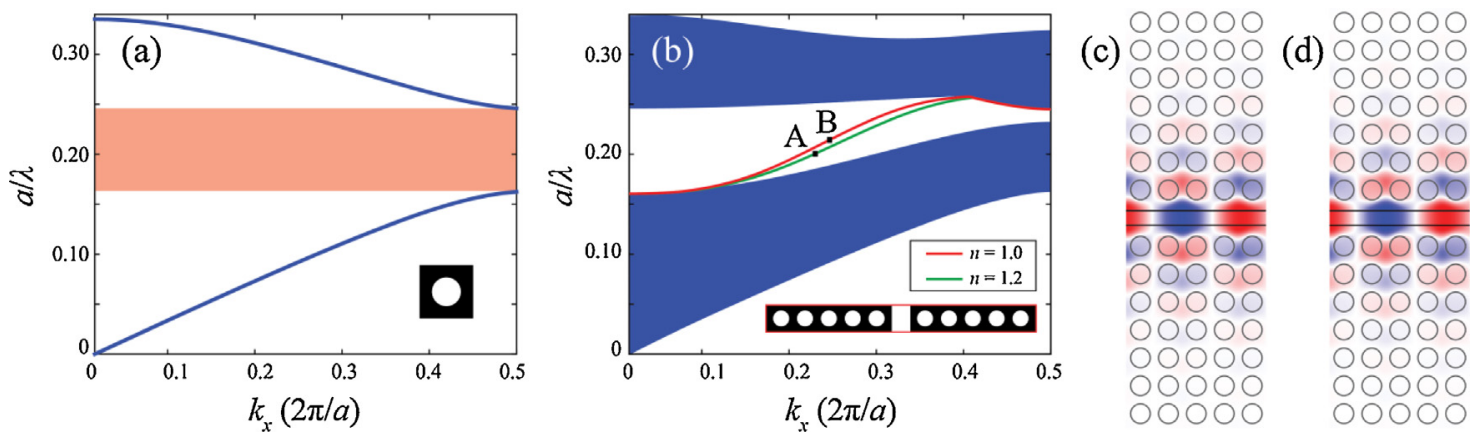

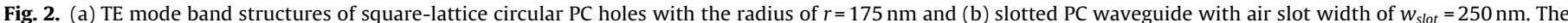

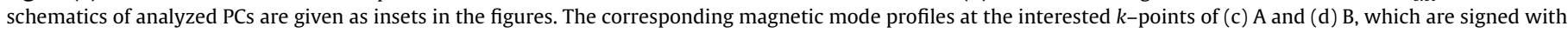
square markers in (b).

line-defects in unperturbed square-lattice PC geometry provides guided multimodes to exist within the photonic bandgap, which is the basic approach to W3-type waveguide design as guiding region of the studied PC based sensor system. The filtering region, however, is formed by introducing an air slot having a width of $w_{\text {slot }}=250 \mathrm{~nm}$ in order to maintain single guided mode at studied frequencies. Dispersion relations for such type of slotted PC waveguide are calculated depending on different refractive indices of analytes and the results are represented in Fig. 2(b). Single guided modes are generated in cases of varying analyte's refractive indices $n=[1.0,1.2]$ in the frequency range of $0.1647-0.2576$ $(a / \lambda)$ and slight frequency shifts occur to lower (dielectric) bands while increasing the analytes' refractive index. That situation may be attributed to a frequency shift at the output transmission peaks, which will be discussed in detail in the next sections. The magnetic field $\left(\mathrm{H}_{\mathrm{z}}\right)$ mode profiles at specific $k$-points $A$ and $\mathrm{B}$ are represented in Fig. 2(c) and (d), respectively. It can be inferred from these figures that despite changing the analytes' refractive indices, a strong field localization occurs in the vicinity of the slotted section for certain frequencies by means of the slotted PC waveguide.

The physical aspect of optical sensing in PC media can be associated with electromagnetic perturbation theory. According to the first order electromagnetic perturbation theory, the spectral shift $\Delta \omega$ due to a small non-dispersive perturbation $\Delta \varepsilon$ in the dielectric function can be expressed as [28]:

$\Delta \omega=-\frac{\omega}{2} \frac{\int d^{3} r \Delta \varepsilon(r)|E(r)|^{2}}{\int d^{3} r \varepsilon(r)|E(r)|^{2}}$

where $\omega$ and $E(r)$ are the angular frequency and mode profile for the dielectric permittivity $\varepsilon$. The perturbed dielectric function, i.e. $\varepsilon(r) \rightarrow \varepsilon(r)+\Delta \varepsilon(r)$, can be expanded into first-order Taylor series for $\Delta \varepsilon<<\varepsilon$. In this case, the small changes in the refractive index of PC media $\Delta n$ can be approximated as $\Delta n \approx \Delta \varepsilon / 2 n$. The filling fraction of energy in perturbed region $f$ can be defined by:

$f=\frac{\int_{s} d r E(r) \cdot D(r)}{\int d r E(r) \cdot D(r)}$

where $D(r)=\varepsilon(r) E(r)$ is the displacement field. The integral in the numerator of Eq. (2) is restricted to the perturbed analyteinfiltration region (denoted by $s$ ), while the denominator is integrated over all PC media. Combining both Eqs. 1 and 2, the spectral shift for small perturbations can be mathematically expressed such as the following:

$\frac{\Delta \omega}{\omega} \cong-\frac{\Delta n}{n} \cdot f$

The $(-)$ sign implies that the mode frequencies tend to decrease while increasing the refractive index of the sample. Changing the variable $\omega$ into $\lambda$, one can reformulate Eq. (3) in terms of the optical wavelength $\lambda$ propagating inside the PC medium:

$\frac{\Delta \lambda}{\Delta n}=f \cdot \frac{\lambda}{n}=S$

It can be deduced from this relation that the sensing capability of PC based sensors directly depends upon the overall filling fraction. Thus, the sensitivity equation, Eq. (4), can be considered as assessment criteria of PC waveguide sensors.

In order to explore light matter interaction and sensing performance of designed PC slab sensor, time domain analyses are carried out by using finite-difference time-domain (FDTD) method [30]. The designed PC slab sensor is illuminated by a broadband Gaussian pulse through the input channel of guiding region and the transmitted beam is detected at the output channel of the 

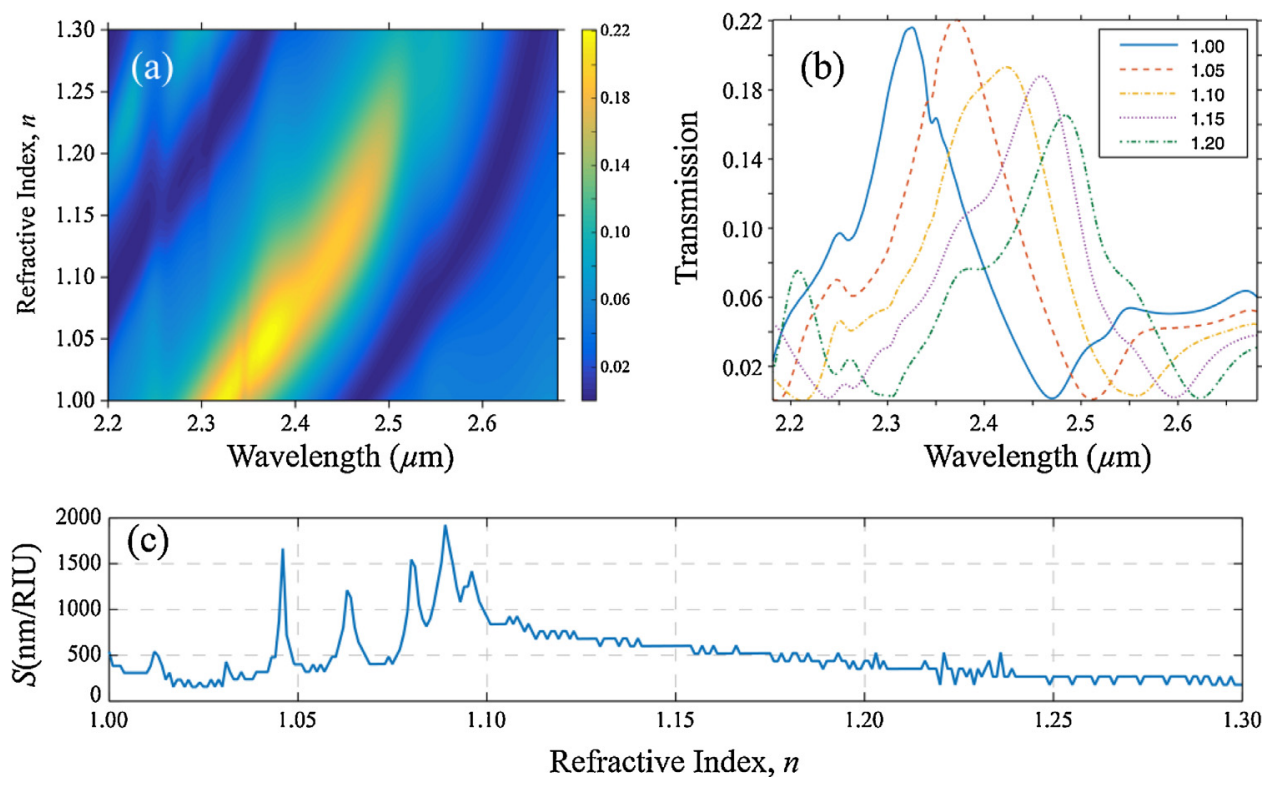

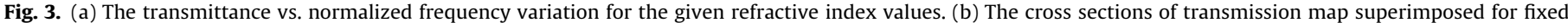
analytes' refractive indices. (c) Corresponding sensitivity variation according to the refractive index changes of analytes.

filtering region. In the following time-domain simulations, the examined analytes that have refractive indices ranging from 1.0 to 1.3 are assumed to be infiltrated inside the T-shaped slot. In this regard, the light-matter interaction behaviour of transmitted wave can be observed from the extracted 2D transmission peaks' map, see Fig. 3(a). As can be understood from Fig. 3(a), light transmission up to $22 \%$ is detected at the output channel and a well-apparent shift in the transmission peaks is observed, which is shown with the yellow region in Fig. 3(a). Especially for incident wavelengths lying in the range of $2.2-2.6 \mu \mathrm{m}$, the output transmission is subjected to a red-shift while increasing the refractive index of infiltrated sample. The transmission cross sections are taken from the map in Fig. 3(a) for fixed analytes' refractive indices of $n=\{1.00,1.05,1.10,1.15,1.20\}$ and superimposed in Fig. 3(b). The red-shift in the transmission peaks indicates that the proposed PC sensor is very sensitive to the refractive index variation of analytes. The sensitivity of the proposed sensor structure $S$ is calculated for the case of $n=1.0-1.3$ and the results are represented in Fig. 3(c). It can be inferred from this figure that the best sensitivity is calculated within the range of $n=1.05-1.10$ with an average value around $S=1040 \mathrm{~nm} / \mathrm{RIU}$ which is a rather good value as compared to PC cavity based optical sensors, where the highest reported value of sensitivity is $S=1500 \mathrm{~nm} / \mathrm{RIU}$ [21]. The overall sensitivity in higher refractive indices $n=1.10-1.30$ is still around the value of $S=500 \mathrm{~nm} / \mathrm{RIU}$, which is fairly good compared to PC waveguide sensors in literature [31-34].

In addition, the magnetic field intensity distributions are calculated for the resonance wavelengths $\lambda=\{2.32 \mu \mathrm{m}, 2.48 \mu \mathrm{m}\}$ at different samples having refractive indices $n=\{1.0,1.2\}$ in order to observe light-matter interaction inside the PC based sensor. Corresponding intensity profiles in the cases of different analytes with $n=\{1.0,1.2\}$ are represented in Fig. 4 (a) and (b), respectively. It can be seen from the figures that the guided light firstly travels through the vertical slot and then, a portion of field power oscillates in vertical direction through the vertical slot. By means of the light feedback mechanism due to back-reflections at the ends, magnetic field enhancement occurs and light is guided inside the horizontal slot. The origin of the wavelength shift may be related with the change in the wavelength resonant Bloch modes inside the vertical slots, which is formulated as $\lambda_{n}=\lambda_{0} / n$. In that relation, $\lambda_{0}$ is the incident wavelength in free space and $n$ is refractive index of inves- tigated analyte. Comparing both cases $n=1.0$ and $n=1.2$, a strong confinement is achieved inside the horizontal slot as can be seen in Fig. 4(a) whereas weak light leakage is obtained in Fig. 4(b) as the effective index of the slot is increased. We should note that, the effect of the vertical slot's dimensions $\left\{s_{x}, s_{y}\right\}$ (see Fig. 1(c) for $s_{x}$ and $s_{y}$ ) on the output field intensity $I$ variation is also investigated. It is found from the variation of $\left\{s_{x}, s_{y}\right\}$ parameters that the dimensions of the vertical part (in $y$-direction, see Fig. 1(c)) of the T-shaped slot have a strong impact on the intensity at the output and that characteristic is described by the following equations:

$$
\begin{aligned}
& I_{\text {out }}=0.0029 \cdot s_{x}-0.01 \quad\left(\mathrm{~s}_{\mathrm{y}}=\text { constant }\right) \\
& I_{\text {out }}=0.064 \cdot e^{-1.08 \cdot S_{y}} \quad\left(\mathrm{~s}_{\mathrm{x}}=\text { constant }\right)
\end{aligned}
$$

According to Eq. (5), changing the vertical size $s_{X}$ leads to a change in the linear field intensity whereas the horizontal size of the vertical slot $s_{y}$ provides an exponential change of the field intensity detected at the output channel. Thus, in the light of above results one can say that underlying sensing mechanism of the T-slot PC design is proven by performed time-domain analyses.

Till now, time-domain analyses are utilized for ideal 2D PC sensors, which are assumed to be infinitely thick. 3D FDTD calculations are also performed to be able to design practical photonic sensors with accurate dimensions. In order to obtain a further quantitative comparison for varying slab thicknesses, the peak wavelengths are calculated for slab thicknesses ranging between $h_{1}=\{0.4 \mu \mathrm{m}, 4.0 \mu \mathrm{m}\}$ with incremental steps equal to $0.1 \mu \mathrm{m}$, and corresponding peak wavelength map is shown in Fig. 5(a). The peak wavelength is defined as the wavelength with the largest transmittance value within the frequency range of interest. Fig. 5(a) shows that a smaller slab thickness yields a lower peak wavelength variation, since the operating guided mode in the band structure will become squeezed as the slab thickness decreases. To compare the 2D ideal case with structures with several different slab thicknesses, the cross sectional profiles taken from Fig. 5(a) are superimposed for $h_{1}=\{1.5 \mu \mathrm{m}, 2.3 \mu \mathrm{m}, 3.1 \mu \mathrm{m}, 3.9 \mu \mathrm{m}\}$ together with $h_{1}=\infty$ (ideal 2D case), and presented in Fig. 5(b). The 2D simulations demonstrate that a relationship between the wavelength and the refractive index of the infiltrated sample exists, which can be numerically fitted by a second-order polynomial function; $\lambda(n)=A \cdot n^{2}+B \cdot n+C$. In this relation, the constant param- 

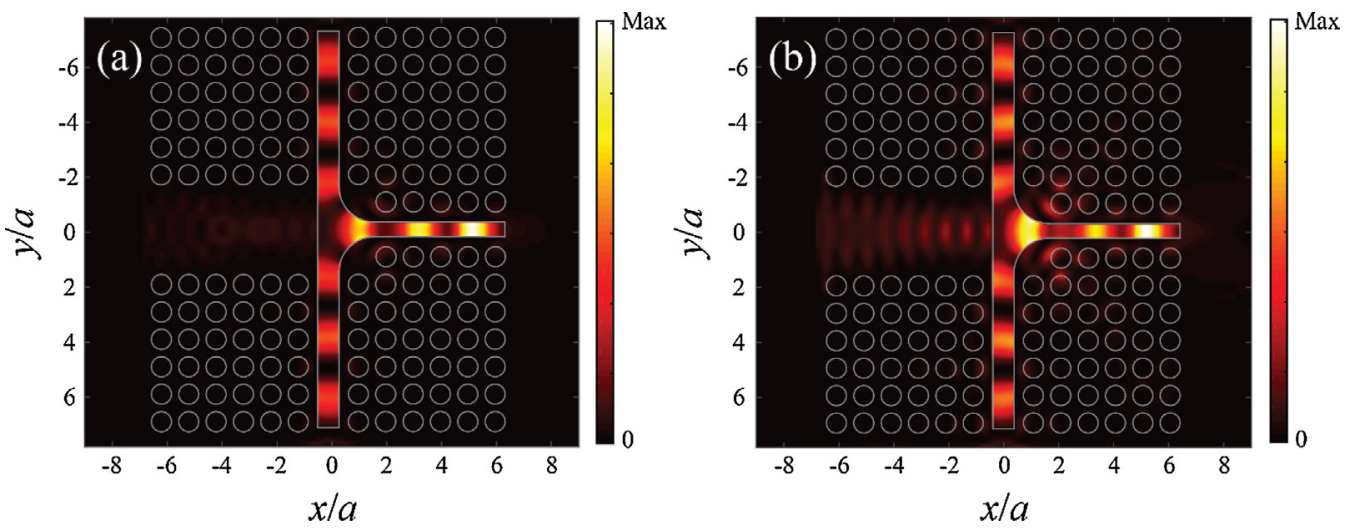

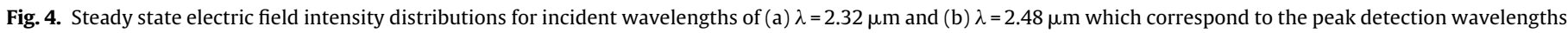

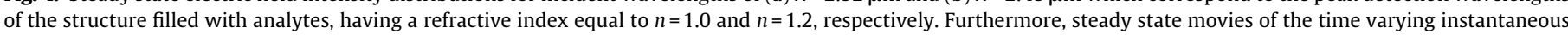
intensity distributions are prepared (see Vis. 1).
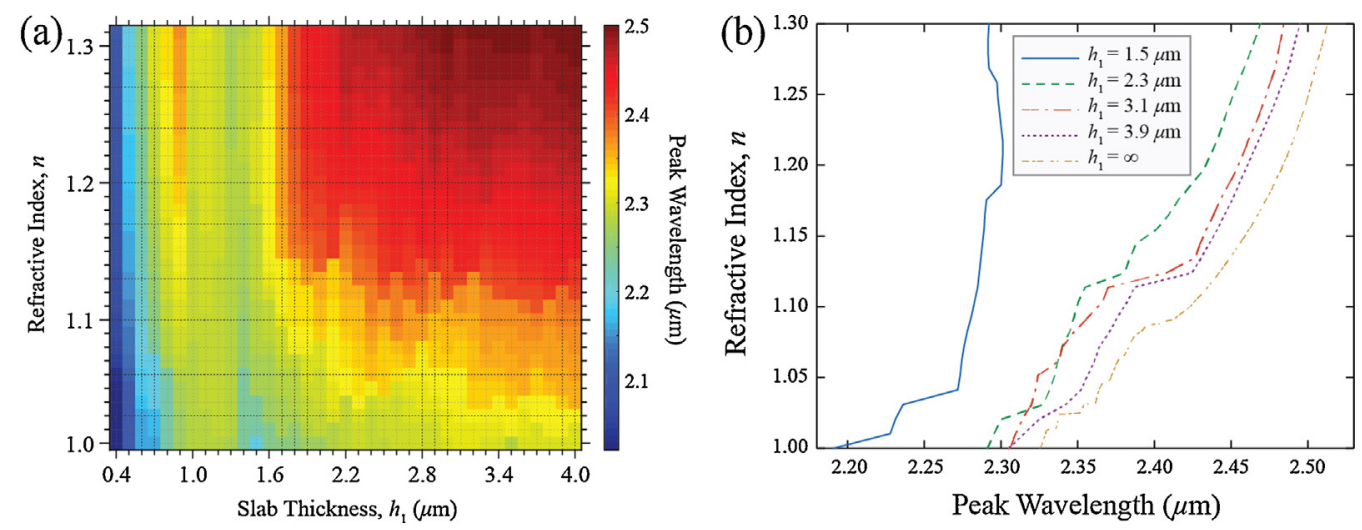

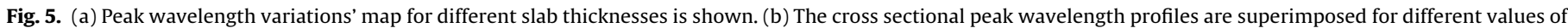
$\mathrm{h}_{1} ; 1.5 \mu \mathrm{m}, 2.3 \mu \mathrm{m}, 3.1 \mu \mathrm{m}, 3.9 \mu \mathrm{m}$ and $\infty$ (2D case).

eters are fixed as $A=-1.90 \mu \mathrm{m}, B=5.03 \mu \mathrm{m}$ and $C=-0.81 \mu \mathrm{m}$. As expected from Fig. 5(b), increasing the slab thicknesses causes the peak wavelength dependency to converge to that of the 2D case. Furthermore, one can also deduce from Fig. 5(a) and (b) that a slab thickness larger than $h_{1}=2.0 \mu \mathrm{m}$ provides a satisfactory peak wavelength sensitivity. Consequently, to meet both the fabrication requirements and the need of a high peak wavelength sensitivity, we have chosen $h_{1}=2.3 \mu \mathrm{m}$ as the optimum slab thickness based on the 3D FDTD analyses.

Fig. 6(a)-(d) are prepared corresponding to the selected thicknesses in Fig. 5(d) and show the variations of the transmittance depending on the refractive index of the sample for slab thicknesses equal to $h_{1}=\{1.5 \mu \mathrm{m}, 2.3 \mu \mathrm{m}, 3.1 \mu \mathrm{m}, 3.9 \mu \mathrm{m}\}$. As can be seen from Fig. 6(a), there is not an apparent wavelength shift in the case of $h_{1}=1.5 \mu \mathrm{m}$ since the propagating mode may not be sufficiently confined inside the PC slab, which weakens the lightmatter interaction providing the transmission peak shifts. On the other hand, as the slab thickness increases, the sensitivity of the peak wavelength dependency onto the refractive index increases as well (see the transmission maps in Fig. 6(b)-(d)).

Since the vertical guiding will rely on the total internal reflection, one should calculate the bandstructure of the PC slab case and determine whether the modes are confined at the slab-air and slab-substrate interface. Fig. 7(a) shows the projected bandstructure for the optimized $2.30 \mu \mathrm{m}$ slab thickness. It can be inferred from this figure that while the waveguide mode is well-confined at the slab-air interface, the confinement at the slab-substrate will be relatively low. However, we performed a loss analysis to quantitatively predict the losses occurring at the slab-substrate. The determined out-of-plane losses into the substrate are within the range of $0.01-0.05 \mathrm{~dB} / \mu \mathrm{m}$ depending on the wavelength and the analyte. It is found that a total substrate loss throughout the whole structure is within the acceptable range of $3-8 \%$. We should note that further losses are also present in the proposed configuration, such as coupling losses, polarization mixing losses due to structural asymmetry, cross sectional mismatch between the source and the structure. Despite such losses we obtained a transmission efficiency around\%20 for the optimized slab thickness (see Fig. 6(b)). Furthermore, the magnetic field profiles of the waveguide mode, given in Fig. 7(b)-(d) for various cross sectional planes, show that the guided mode is mainly concentrated at the slot region, allowing sufficient interaction between the analyte and the propagating wave.

Another key point that should be taken into account in a realistic slab PC case is that the waveguide mode dispersion will differ from its 2D counterpart. Due to the effective index differences between the slab and 2D case, the waveguide mode will become squeezed and the refractive index sensitivity, which depends on the spectral shift of the mode dispersion, is expected to decrease. Fig. 8 shows the refractive index sensitivity analysis of the slab PC case. The mean sensitivity values are calculated as $530 \mathrm{~nm} / \mathrm{RIU}$ and $390 \mathrm{~nm} / \mathrm{RIU}$ for refractive indices in the range of 1.10-1.25 and $1.00-1.30$, respectively.

\section{Potential fabrication platform for proposed PC based sensor}

The fabrication of the proposed T-slotted PC slab sensor can be realized on a silicon-on-insulator (SOI) substrate utilizing electron- 

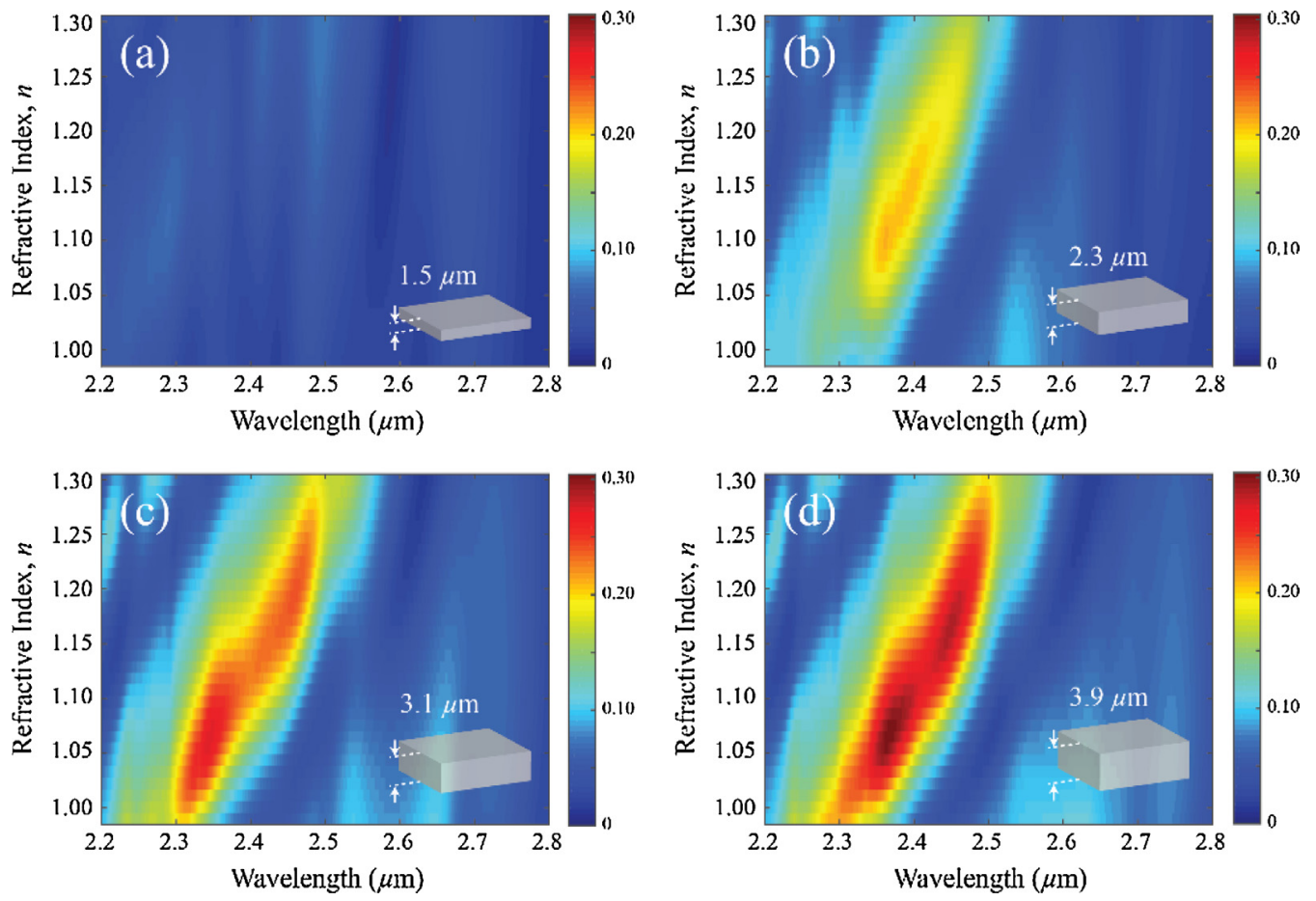

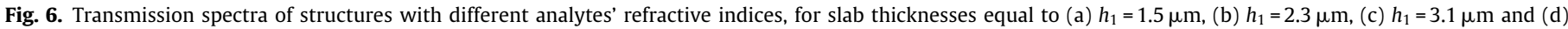
$h_{1}=3.9 \mu \mathrm{m}$.
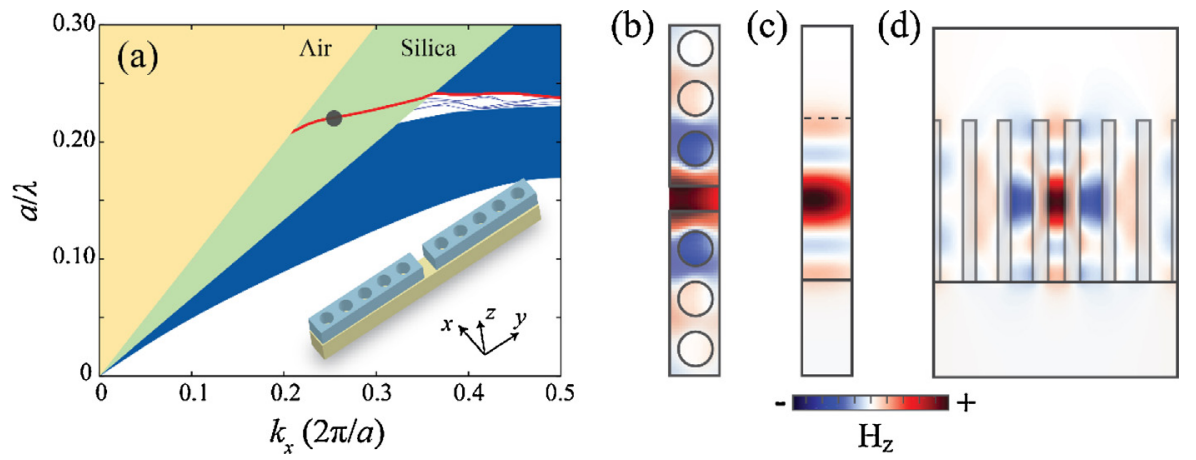

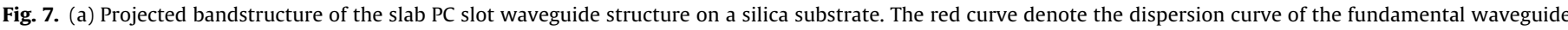

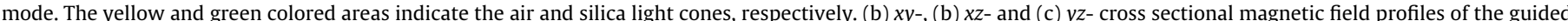

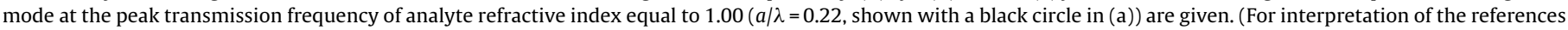
to color in this figure legend, the reader is referred to the web version of this article.)

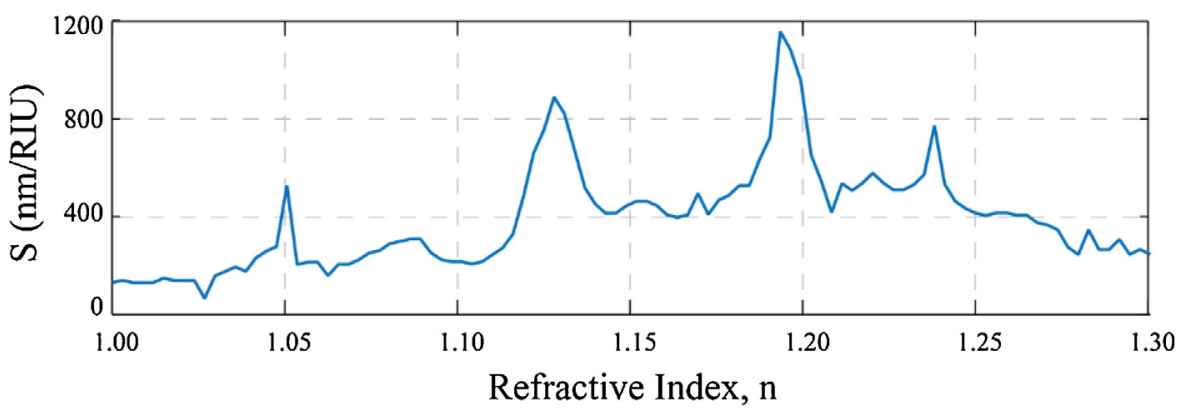

Fig. 8. Refractive index sensitivity variation of the slab PC structure.

beam lithography (EBL) tool. Among the simulated slotted PC waveguides, the most promising structure providing highest sensitivity has $h_{1}=3.9 \mu \mathrm{m}$ and $r=175 \mathrm{~nm}$, giving rise to an aspect ratio of $\sim 11$, where aspect ratio is defined as $h_{1} /(2 r)$. A possible fabrication flow is given in Fig. 9. A good choice of e-beam resist for such high-aspect-ratio PC holes is ZEP 520, which can also be used as a mask during the dry etching process after it is exposed with e-beam followed by development in ZED-N50. Based on the etch rate selectivity between silicon and ZEP 520 reported in Ref. [35], a $300 \mathrm{~nm}$-thick layer of ZEP 520 can serve as a mask to etch 3.9- 


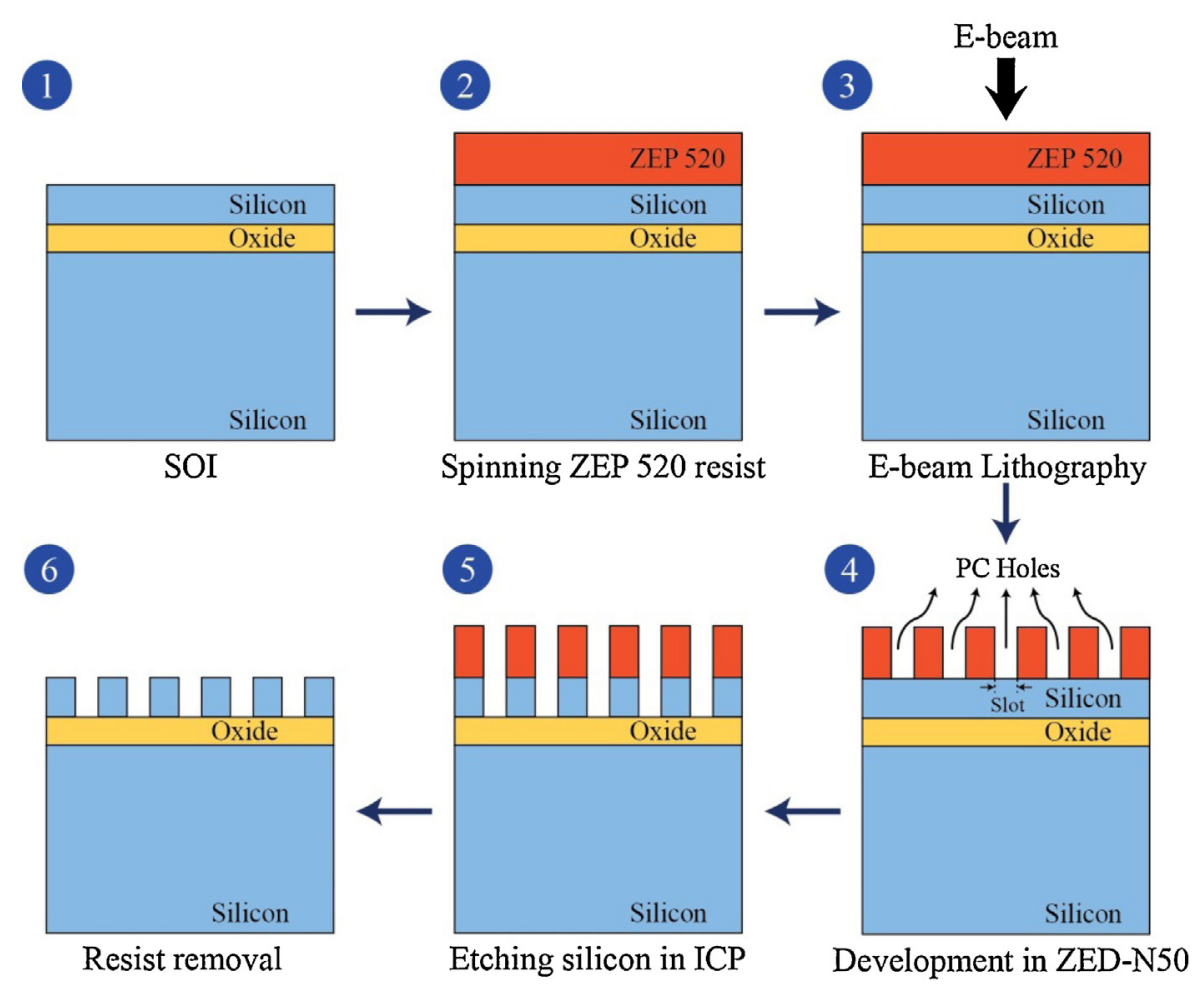

Fig. 9. The potential fabrication flow of the simulated slotted PC waveguides.

$\mu \mathrm{m}$-thick silicon through holes that are $350 \mathrm{~nm}$ in diameter. It is essential to ensure that the etching is anisotropic, to be able to obtain high-aspect-ratio PC holes with vertical sidewalls. Therefore, inductively-coupled plasma (ICP)-type of dry etcher can be used to etch silicon in a gas environment of $\mathrm{SF}_{6}$ and $\mathrm{C}_{4} \mathrm{~F}_{8} . \mathrm{SF}_{6}$ will etch the silicon down to the oxide surface while $\mathrm{C}_{4} \mathrm{~F}_{8}$ will provide anisotropic etching through passivation of the sidewalls [36].

The optical characterization of the fabricated structure can be carried out via optical transmission measurements by introducing several different analytes into the air slot of the waveguide. The polydimethylsiloxane (PDMS)-based microfluidic channels can be fabricated and bonded on top of the slotted waveguides as reported in Ref. 20, so as to deliver different analytes into the nanoscale slot. The opposite facets of the waveguide, where light will couple inand out-via tapered fibers residing on 6-axis nano-positioning flexure stages, should be mirror polished for the purpose of reducing the scattering losses [37]. The alignment of the fiber tips to the input and output facets of the waveguide can be controlled by mounting the slotted PC waveguides under a magnification system. As illustrated in Fig. 10, the input light can be introduced from a broadband MIR light source to the input facet of the slotted waveguide through a polarization-maintaining tapered fiber that will allow controlling the input polarization. The transmitted TE-polarized light will be coupled into another tapered fiber and sent into an optical spectrum analyser with a built-in monochromator and photodetector through the fiber.

The basic detection methods in optical bio-chemical sensors can be listed as: flourescence-based and label-free detection. In the first method, flourescent labels are utilized in order to identify the presence of target molecules. Nevertheless, such a flourescence detection technique may require isolation of target molecules as well as additional labelling procedures. On the other hand, labelfree detection technique does not require the modifications of target molecules, which provides easier and cheaper bio-chemical sensing. Refractive-index based sensors are amongst label-free optical sensors. Most of the label-free optical sensors are designed to work at the telecommunication wavelengths or mid infrared wavelengths. Such type of optical sensors has different structural properties and is made of a diverse combination of materials for sensing various objects such as gases, solutions and organic particles. For instance, a PC microring resonator [38], a PC nano slotted parallel cavity [39] and a PC nanobeam cavity [40] structures have already been introduced. The common properties of such sensors are performing refractive index based sensing and operating at the telecommunication wavelength of $1550 \mathrm{~nm}$. Reported sensitivity values of these sensors are below $460 \mathrm{~nm} / \mathrm{RIU}$, which is less than the presented PC slab sensor case. In most of the recent studies, the PC based sensors that operate at MIR wavelengths are selected to be Si-based due to being suitable for organic and inorganic chemicals or liquids. For example, a sensor structure consists of parallel PC waveguides has been designed for fluid sensing and chemical imaging in Ref. [41]. Moreover, a holey and a slotted PC waveguide structures that are based on $\mathrm{Si}$ are designed to detect Triethylphosphate at MIR wavelength [42].

\section{Conclusion}

In this study, we presented an optical refractive index sensor design for mid-IR photonics, with a special emphasis on air-slot photonic crystal waveguide to have a strong on-chip field confinement. Numerical methods based on finite-difference time-domain and plane-wave expansion method are performed to evaluate the refractive index detection sensitivity of the designed sensor. In order to correlate the sensor performance in terms of strong light confinement parameter, analytical derivations are presented. The best calculated sensitivity of the proposed T-slotted PC sensor is around $1040 \mathrm{~nm} / \mathrm{RIU}$ in the case of analytes' refractive indices ranging from $n=1.05$ to $n=1.10$. Besides, an overall sensitivity is calculated to be around $500 \mathrm{~nm} / \mathrm{RIU}$ for the case of higher refractive indices of analytes $n=1.10-1.30$. Additionally, for a realistic PC slab structure we determined an average refractive index sensitivity of $530 \mathrm{~nm} / \mathrm{RIU}$ within the range of $\mathrm{n}=1.10-1.25$ and an average 


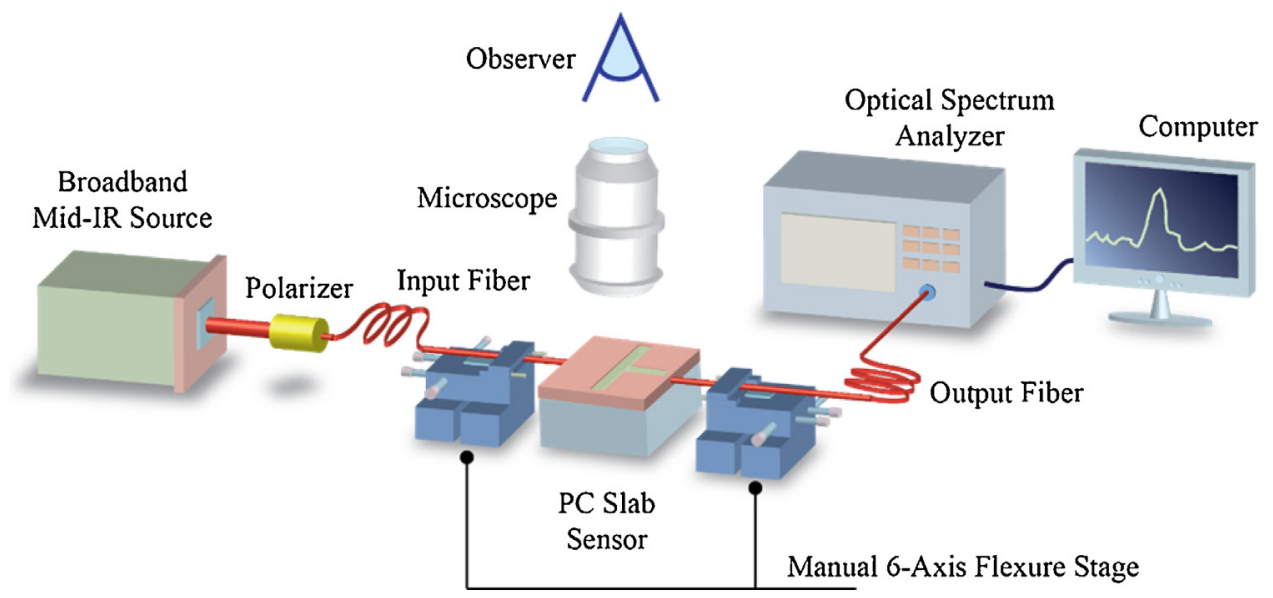

Fig. 10. The potential optical characterization setup for the designed refractive index PC slab sensor.

sensitivity of $390 \mathrm{~nm} / \mathrm{RIU}$ within the range of $\mathrm{n}=1.00-1.30$.Possible fabrication and characterization methods are provided in the manuscript. The reported results show that the highly sensitive refractive index sensing in the mid-IR region is feasible as long as effort to optimize the structural parameters of the photonic platform is conducted.

\section{Acknowledgments}

M.T. gratefully acknowledges the support of the TED University Scientific Research Project with Project No: 16A303. H.K. acknowledges partial support from the Turkish Academy of Sciences. M.T., Z.H., H.K. and K.S. also acknowledge partial support of NATO SPS985048 grant.

\section{Appendix A. Supplementary data}

Supplementary data associated with this article can be found, in the online version, at http://dx.doi.org/10.1016/j.snb.2017.02.016.

\section{References}

[1] B. Troia, A. Paolicelli, F. De Leonardis, V.M.N. Passaro, Photonic Crystals for Optical Sensing: A Review, INTECH Open Access Publisher, 2013, pp. 242-295.

[2] C. Ciminelli, F. Dell'Olio, D. Conteduca, C.M. Campanella, M.N. Armenise, High performance SOI microring resonator for biochemical sensing, Opt. Laser Technol. 59 (2014) 60-67.

[3] C. Wu, A.B. Khanikaev, R. Adato, N. Arju, A.A. Yanik, H. Altug, G. Shvets, Fano-resonant asymmetric metamaterials for ultrasensitive spectroscopy and identification of molecular monolayers, Nat. Mater. 11 (2011) 69-75.

[4] Q. Liu, X. Tu, K.W. Kim, J.S. Kee, Y. Shin, K. Han, Y.J. Yoon, G.Q. Lo, M.K. Park, Highly sensitive Mach-Zehnder interferometer biosensor based on silicon nitride slot waveguide, Sens. Actuators B Chem. 188 (2013) 681-688.

[5] C. Kang, S.M. Weiss, Photonic crystal with multiple-hole defect for sensor applications, Opt. Express 16 (2008) 18188-18193.

[6] L. Shi, A. Kabashin, M. Skorobogatiy, Spectral, amplitude and phase sensitivity of a plasmonic gas sensor in a metallic photonic crystal slab geometry: comparison of the near and far field phase detection strategies, Sens. Actuators B Chem. 143 (2009) 76-86.

[7] A.V. Kabashin, S. Patskovsky, A.N. Grigorenko, Phase and amplitude sensitivities in surface plasmon resonance bio and chemical sensing, Opt. Express 17 (2009) 21191-21204.

[8] E. Yablonovitch, Inhibited spontaneous emission in solid-state physics and electronics, Phys. Rev. Lett. 58 (1987) 2059-2062.

[9] H. Lin, Y. Zou, J. Hu, Double resonance 1-D photonic crystal cavities for single-molecule mid-infrared photothermal spectroscopy: theory and design, Opt. Lett. 37 (2012) 1304-1306.

[10] J. Jágerská, H. Zhang, Z. Diao, N. Le Thomas, R. Houdré, Refractive index sensing with an air-slot photonic crystal nanocavity, Opt. Lett. 35 (2010) 2523.

[11] S. Chakravarty, W.-C. Lai, X. W, C. Lin, R.T. Chen, Photonic crystal slot waveguide spectrometer for the detection of methane. Proc. SPIE 7941, Integr. Opt.: Devices Mater. Technol. XV (2011) 79410 K.

[12] K.V. Shanthi, S. Robinson, Two-dimensional photonic crystal based sensor for pressure sensing, Photonic Sens. 4 (2014) 248-253.
[13] T. van Leest, J. Caro, Cavity-enhanced optical trapping of bacteria using a silicon photonic crystal, Lab. Chip 13 (2013) 4358-4365.

[14] F.L. Hsiao, C. Lee, Computational study of photonic crystals nano-ring resonator for biochemical sensing, IEEE Sens. J. 10 (2010) 1185-1191.

[15] Y.-N. Zhang, Y. Zhao, H.-F. Hu, Miniature photonic crystal cavity sensor for simultaneous measurement of liquid concentration and temperature, Sens. Actuators B. Chem. 216 (2015) 563-571.

[16] A.A. Yanik, A.E. Cetin, M. Huang, A. Artar, S.H. Mousavi, A. Khanikaev, J. Connor, G. Shvets, H. Altug, Ultrasensitive plasmonic fano sensor enables seeing protein monolayers with naked eye, IEEE Photonic Soc 24th Annu. Meet. PHO 2011 (2011) 425-426

[17] S.M. Nalawade, S.S. Harnol, H.V. Thakur, Temperature and strain independent modal interferometric torsion sensor using photonic crystal fiber, IEEE Sens. J. 12 (2012) 2614-2615

[18] H. Qu, M. Skorobogatiy, Resonant bio- and chemical sensors using low-refractive-index-contrast liquid-core Bragg fibers, Sens. Actuators B Chem. 161 (2012) 261-268.

[19] M.G. Scullion, A. Di Falco, T.F. Krauss, Slotted photonic crystal cavities with integrated microfluidics for biosensing applications, Biosens. Bioelectron. 27 (2011) 101-105.

[20] M.G. Scullion, T.F. Krauss, A. Di Falco, Slotted photonic crystal sensors, Sensors (Switzerland) 13 (2013) 3675-3710.

[21] Y.-N. Zhang, Y. Zhao, R.-Q. Lv, A review for optical sensors based on photonic crystal cavities, Sens. Actuators A Phys. 233 (2015) 374-389.

[22] R.W. Waynant, I.K. Ilev, I. Gannot, Mid-infrared laser applications in medicine and biology, Philos. Trans. R. Soc. A Math. Phys. Eng. Sci. 359 (2001) 635-644.

[23] Y. Zou, S. Chakravarty, P. Wray, R.T. Chen, Mid-infrared holey and slotted photonic crystal waveguides in silicon-on-sapphire for chemical warfare simulant detection, Sens. Actuators B Chem. 221 (2015) 1094-1103.

[24] S.S. Kim, C. Young, B. Mizaikoff, Miniaturized mid-infrared sensor technologies, Anal. Bioanal. Chem. 390 (2008) 231-237.

[25] R. Shankar, R. Leijssen, I. Bulu, M. Lončar, Mid-infrared photonic crystal cavities in silicon, Opt. Express 19 (2011) 5579-5586.

[26] R. Soref, Mid-infrared photonics in silicon and germanium, Nat. Photonics 4 (2010) 495-497.

[27] C. Babayiğit, M. Turduev, I.H. Giden, E. Bor, H. Kurt, T-shape slotted photonic crystal based sensor with high sensitivity, in: 18th International Conference on Transparent Optical Networks (ICTON), Trento, Italy, 2016, pp. 1-4.

[28] J.D. Joannopoulos, R.D. Meade, J.N. Winn, Photonic Crystals: Molding the Flow of Light, University Press, Princeton, 1995.

[29] S. Johnson, J.D. Joannopoulos, Block-iterative frequency-domain methods for Maxwell's equations in a planewave basis, Opt. Express 8 (2001) 173.

[30] FDTD Solutions, (version 8.16) from Lumerical Solutions Inc., http://www lumerical.com.

[31] N. Skivesen, A. Têtu, M. Kristensen, J. Kjems, L.H. Frandsen, P.I. Borel, Photonic-crystal waveguide biosensor, Opt. Express 15 (2007) 3169-3176.

[32] Y. Liu, H.W.M. Salemink, Photonic crystal-based all-optical on-chip sensor, Opt. Express 20 (2012) 19912-19920.

[33] H. Kurt, M.N. Erim, N. Erim, Various photonic crystal bio-sensor configurations based on optical surface modes, Sens. Actuators B Chem. 165 (2012) 68-75.

[34] C.A. Barrios, K.B. Gylfason, B. Sánchez, A. Griol, H. Sohlström, M. Holgado, R. Casquel, Slot-waveguide biochemical sensor, Opt. Lett. 32 (2007) 3080.

[35] Y. Xu, H.-B. Sun, J.-Y. Ye, S. Matsuo, H. Misawa, Fabrication and direct transmission measurement of high-aspect-ratio two-dimensional silicon-based photonic crystal chips, J. Opt. Soc. Am. B 18 (2001) 1084

[36] K. Solehmainen, T. Aalto, J. Dekker, M. Kapulainen, M. Harjanne, K. Kukli, P. Heimala, K. Kolari, M. Leskelä, Dry-etched silicon-on-insulator waveguides with low propagation and fiber-coupling losses, J. Lightwave Technol. 23 (2005) 3875-3880. 
[37] Y. Li, A. Bhattacharyya, C. Thomidis, T.D. Moustakas, R. Paiella, Nonlinear optical waveguides based on near-infrared intersubband transitions in GaN/AlN quantum wells, Opt. Express 15 (2007) 5860-5865.

[38] D. Urbonas, A. Balcytis, K. Vaskevicius, M. Gabalis, R. Petruskevicius, Air and dielectric bands photonic crystal microringresonator for refractive index sensing, Opt. Lett. 41 (2016) 3655-3658.

[39] D. Yang, H. Tian, Y. Ji, Photonic crystal nanoslotted parallel quadrabeam integrated cavity for refractive index sensing with high figure of merit, Phot. Nano. Fund. Appl. 15 (2014) 124-129.

[40] T. Lin, X. Zhang, G. Zhou, C.F. Siong, J. Deng, Design of an ultra-compact slotted photonic crystal nanobeam cavity for biosensing, J. Opt. Soc. Am. B 32 (2015) 1788-1791.

[41] V.M. Lavchiev, B. Jakoby, U. Hedenig, T. Grille, J.M.R. Kirkbride, G.A.D. Ritchie, M-line spectroscopy on mid-infrared Si photonic crystals for fluid sensing and chemical imaging, Opt. Express 24 (2016) 262-271.

[42] Y. Zou, S. Chakravarty, P. Wray, R.T. Chen, Mid-infrared holey and slotted photonic crystal waveguides in silicon-on-sapphire for chemical warfare simulant detection, Sens. Actuators B 221 (2015) 1094-1103.

\section{Biographies}

Mirbek Turduev received the Qualified Engineer from Control Engineering Department of the Kyrgyz State Technical University, Kyrgyzstan, Bishkek, in 2006, the M.S. and Ph.D. degree from the Electrical and Electronic Engineering Department of TOBB University of Economics and Technology in 2010 and 2015, respectively. Dr. Turduev in his doctoral dissertation investigated and characterized new photonic designs for efficiently light manipulation, beam steering, beam splitting, wavelength de-multiplexing, light focusing, higher order mode converging, limited diffraction beam generation and asymmetric light transmission purposes. His published works include one book chapter, more than 20 refereed scientific articles, one invited paper and more than 30 international conference proceedings. Dr. Turduev has worked in various scientific projects supported by European Union 6th Framework program and Scientific and Technological Research Council of Turkey (TÜBITAK) as an expert researcher. Since September 2015, he has been with the TED University, Ankara as an Assistant Professor Doctor in electrical and electronics engineering department. His present research examines light matter interaction phenomena in the field of photonics including the design and analysis of photonic devices for the realization of sub-wavelength scale optical devices, graded index optics, high-resolution imaging, polarization insensitive devices, gain loss modulated structures, and optical biosensors. Moreover, he is a Co-Founder of Photonics Networks Research Group at the TED University.

Ibrahim Halil Giden is a PhD. Student at TOBB University of Economics and Technology in Ankara, TURKEY and is a member of Nanophotonics research group advised by Prof. Hamza Kurt since 2011. He has received BS degree from Middle East Technical University, Department of Electrical and Electronics Engineering and MSc. Degree from TOBB University of Economics and Technology in Ankara, TURKEY. He has published 20 peer-reviewed scientific papers including one invited papers. He has given contributed and invited presentations and published more than 17 conference abstracts and proceedings. His main research interests include nanophotonics, slow light structures, graded index optics, optical cavities/waveguides, biosensors and photonic crystals.

Ceren Babayigit is an undergraduate student at TED University, Department of Electrical and Electronics Engineering. Also she is a member of Photonic Networks Research Group.

Zeki Hayran received the B.S. degree from the Department of Electrical and Electronics Engineering at the TOBB University of Economics and Technology, Ankara, Turkey, in 2015. He is currently working toward the M.S. degree in Electrical and Electronics Engineering at the TOBB University of Economics and Technology, Ankara, Turkey.
Emre Bor received his B.Sc. degree from Gazi University, Department of Electrical and Electronics Engineering in 2013. He received his M.Sc. degree from TOBB University of Economics and Technology, Department of Electrical and Electronics Engineering in 2016. He pursues his Ph.D. degree studies at TOBB University of Economics and Technology, Department of Electrical and Electronics Engineering. His research interests include nanophotonics and photonic crystals. Since September 2014, he has been working as a research and teaching assistant at TED University, Department of Electrical and Electronics Engineering.

Cicek Boztug received her B.S. degree in Physics from the Middle East Technical University (METU), Ankara, Turkey in 2006. She received her M.S. degree from the University of Connecticut (UCONN), Storrs, CT, USA, in 2009, and her Ph.D. degree from the Boston University (BU), Boston, MA, USA, in 2014, both in Electrical Engineering. She has joined to TED University (TEDU), Ankara, Turkey, in 2014 as an assistant professor. Her research interests include development of flexible optoelectronic devices, strained-germanium based photonic devices, and group-IV photonics for the applications of biochemical sensing and optical communication.

Hamza Kurt received the B.S. degree from Middle East Technical University (METU), Ankara, Turkey, in 2000, the M.S. degree from the University of Southern California (USC), Los Angeles, CA, USA, in 2002, and the Ph.D. degree from the Georgia Institute of Technology (Georgia Tech), Atlanta, GA, USA, in 2006, all in electrical and electronics engineering. He was a Research Fellow with the Cedars-Sinai Medical Center, Bio-photonics Research Lab, Los Angeles, from 2001 to 2002. He was a Post-Doctoral Research Fellow with Georgia Tech for a short period and then he has spent a year with the Institut dOptique Graduate School, Paris, France, as a Post-Doctoral Scientist. Since December 2007, he has been with the TOBB University of Economics and Technology, Ankara and promoted to full-professor level in 2015. He has published more than 65 peer-reviewed scientific papers including two invited papers and six single-author papers. He has given contributed and invited presentations and published more than 60 conference abstracts and proceedings. His current research interests include nanophotonics including the design and analysis of nanophotonic materials and media for the realization of wavelength and sub-wavelength scale optical elements, slow light structures, graded index optics, high-resolution imaging, polarization insensitive devices, optical cavities/waveguides, and optical biosensors. He received the Outstanding Faculty of the Year Award 2010, TOBB University and Turkish Academy of Sciences Distinguished Young Scientist Award (TUBA-GEBIP) 2010. Prof. Kurt is a member of the Optical Society of America, IEEE Photonics Society and associate member of Turkish Academy of Sciences. He is a member of editorial board of Advanced Electromagnetics (AEM) journal and director of nanophotonics research group at TOBB University in Ankara.

Kestutis Staliunas received the Graduation degree in theoretical physics, the Ph.D. degree in physics, and the Habilitation degree in physics from Vilnius University, Vilnius, Lithuania, in 1985, 1989, and 2001, respectively. He was an A.v. Humboldt Fellow with Physikalisch-Technische Bundesantstalt (PTB), Braunschweig, Germany during 1991-1992. Between 1993 and 2003, he was a Senior Research Fellow in Braunschweig PTB and the University of Hanover researching on nonlinear pattern formation in lasers (optical vortices, spatial solitons) and in Bose condensates. Since 2004, he has been ICREA Research Professor with the Universitat Polit'ecnica de Catalunya, Barcelona, Spain. He is also the Head of research group on lasers, photonic and sonic crystals, nonlinear laser dynamics. He has published 200 articles in scientific journals He has given 500 presentations in conferences and 50 invited presentations. He holds two patents. He has published "Transverse patterns in nonlinear optical resonators" in Springer Tracts in Modern Physics, vol. 183, 2003. He has directed (or codirected) 11 Ph.D. projects. 